\title{
Supravalvular Aortic Stenosis Caused by a Familial Chromosome 7 Inversion Disrupting the ELN Gene Uncovered by Whole-Genome Sequencing
}

\author{
Linda Pons $^{\mathrm{a}, \mathrm{b}}$ Patrice Bouvagnet ${ }^{c, d} \quad$ Mohamed Bakloul $^{\mathrm{e}}$ Sylvie Di Filippo ${ }^{\mathrm{d}, \mathrm{e}}$ \\ Adrien Buisson $^{\mathrm{a}}$ Nicolas Chatron ${ }^{\mathrm{a}, \mathrm{b}, \mathrm{d}}$ Audrey Labalme ${ }^{\mathrm{a}}$ Olivier Metton $^{\mathrm{e}}$ \\ Julia Mitchell $^{\mathrm{e}}$ Flavie Diguet ${ }^{\mathrm{a}, \mathrm{b}}$ Pierre-Antoine Rollat-Farnier ${ }^{\mathrm{a}}$ \\ Damien Sanlaville ${ }^{a, b, d}$ Caroline Schluth-Bolard ${ }^{a, b, d}$

\begin{abstract}
a Laboratoire de Cytogénétique Constitutionnelle, Service de Génétique, Centre de Biologie et de Pathologie Est, Hospices Civils de Lyon, Bron, France; b GENDEV Team, Lyon Neuroscience Research Center, CNRS UMR 5292 , INSERM U1028, UCBL1, Bron, France; 'Laboratoire de Cardiogénétique, Centre de Biologie et de Pathologie Est,

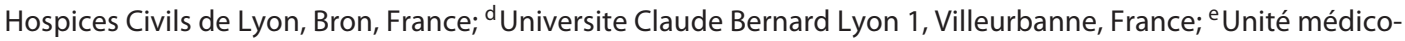
chirurgicale des Cardiopathies Congénitales Adultes et Enfants, Hôpital Louis Pradel, Hospices Civils de Lyon, Bron, France
\end{abstract}

\section{Keywords}

Elastin $\cdot E L N \cdot$ Paracentric inversion · Supravalvular aortic stenosis - Whole-genome sequencing

\begin{abstract}
Apparently, balanced chromosomal rearrangements usually have no phenotypic consequences for the carrier. However, in some cases, they may be associated with an abnormal phenotype. We report herein the case of a 4-year-old boy presenting with clinically isolated supravalvular aortic stenosis (SVAS). No chromosomal imbalance was detected by array CGH. The karyotype showed a balanced paracentric chromosome 7 inversion. Breakpoint characterization using paired-end whole-genome sequencing (WGS) revealed an ELN gene disruption in intron 1, accounting for the phenotype. Family study showed that the inversion was inherited, with incomplete penetrance. To our knowledge, this is the first case of a disruption of the ELN gene characterized by WGS. It contributes to refine the genotype-phenotype correlation in $E L N$ disruption. Although this disruption is a rare
\end{abstract}

etiology of SVAS, it cannot be detected by the diagnostic tests usually performed, such as array CGH or sequencing methods (Sanger, panel, or exome sequencing). With the future perspective of WGS as a diagnostic tool, it will be important to include a structural variation analysis in order to detect balanced rearrangements and gene disruption.

(c) 2019 S. Karger AG, Basel

\section{Introduction}

Supravalvular aortic stenosis (SVAS; MIM 185500), characterized by the congenital narrowing of the aortic lumen, is a rare cardiac anomaly with an incidence of approximately 1:25,000 births [Metcalfe et al., 2000]. SVAS is the major feature of the elastin arteriopathy, related to haploinsufficiency of the ELN gene, located in 7q11.23, which encodes the elastin protein [Merla et al., 2012]. SVAS is a classical feature of Williams-Beuren syndrome (WBS; MIM 194050), due to a recurrent microdeletion in 7q11.23 encompassing the ELN gene [Pober, 2010]. This

\section{KARGER}

(c) 2019 S. Karger AG, Basel

E-Mail karger@karger.com

www.karger.com/msy
Linda Pons

Laboratoire de Cytogénétique Constitutionnelle, Service de Génétique

Centre de Biologie et de Pathologie Est

59, boulevard Pinel, FR-69677 Bron Cedex (France)

E-Mail linda.pons@chu-lyon.fr 
syndrome is characterized by intellectual disability, hypercalcemia, impaired social interactions, facial dysmorphism, and elastin arteriopathy. SVAS may also be isolated and transmitted in an autosomal dominant manner with incomplete penetrance and variable expressivity. ELN Sanger sequencing identifies molecular alterations in approximately $50 \%$ of the cases [Metcalfe et al., 2000]. Apparently, balanced chromosomal rearrangements usually have no phenotypic consequences for the carrier. However, in some cases, they may be associated with an abnormal phenotype due to a cryptic imbalance or a disturbance of gene expression at the breakpoints. Mapping of breakpoints using classical methods such as FISH or Southern blotting has scarcely been used in diagnostic settings. More recently, next-generation sequencing was found to be a rapid and efficient method to characterize the breakpoints at molecular level, providing information about their mechanisms and identifying disease-causing genes [Schluth-Bolard et al., 2013]. We report herein the case of a 4-year-old boy presenting with clinically isolated SVAS associated with an inherited paracentric chromosome 7 inversion. Charaterization of this inversion by paired-end whole-genome sequencing (WGS) revealed ELN disruption.

\section{Patients and Methods}

\section{Clinical Reports}

The index case is a 4-year-old boy (III:1, Fig. 1A) presenting with clinically isolated SVAS. He is the first born of a twin bichorial biamniotic pregnancy, an eutrophic premature at 32 weeks of amenorrhea. The neonatal period was uneventful. A rough aortic murmur 3/6 was found at 11 months of age, fully symptom free. The echocardiography showed an isolated SVAS measured at 5.7 $\mathrm{mm}$ with a maximal gradient at $118 \mathrm{mmHg}$ and a mean gradient at $64 \mathrm{mmHg}$. The prestenotic aorta was measured at $15.8 \mathrm{~mm}$ for a poststenotic aorta at $13.8 \mathrm{~mm}$. The left ventricle was not dilated, normokinetic, but hypertrophied, without mitral insufficiency. The aortic valve appeared asymmetric but tricuspid, without valvular stenosis. There was a micro aortic insufficiency $0.5 / 4$ and a common origin of the innominate artery and the left primary carotid artery. The coronary arteries looked anatomically normal. A scan showed an SVAS of approximately $50 \%$ of the diameter without further anomalies of the thoracic aorta. He underwent a patch aortoplasty and plasty of the Valsalva sinuses at 32 months of age, without complications. Functional result was excellent. The last echocardiography, at 4 years of age, found no persistent SVAS, but an acceleration of the flow in the tubular aorta $(2.6 \mathrm{~m} / \mathrm{s})$ with stable mild aortic insufficiency. Psychomotor development was normal, with regular schooling. Growth was normal as well, with a weight of $15 \mathrm{~kg}(-1 \mathrm{SD})$ and a height of $106 \mathrm{~cm}$ (average) at the last observation, 4 years of age. Blood pressure was normal. Serum calcium level was normal. There were no dysmorphic features suggestive of WBS. The twin sister of the index case (III:2, Fig. 1A) is healthy. Both of the boy's parents (II:5 and II:4) have a heart murmur, with normal echocardiographic values as well as the maternal grandparents and 2 maternal aunts. Likewise among 3 paternal aunts, the eldest one (II:1) was monitored since infancy for an isolated symptom-free SVAS. The middle (II:2) is healthy, and the youngest (II:3) has a heart murmur, with normal echocardiographic values. The paternal grandmother $(\mathrm{I}: 2)$ is healthy. The paternal grandfather (I:1) has a mitral valve prolapse in the context of a ballooned dystrophic mitral valve, with no left ventricle impact.

\section{Materials and Methods}

Array CGH was performed on extracted DNA from the lymphocytes of the index case with the QIAmp DNA Blood Midi kit (Qiagen, Vento, The Netherlands), with a custom $244 \mathrm{~K}$ oligonucleotide microarray enriched at the 7q11.23 locus (SurePrint Custom-HD CGH Microarray, 1X244KK, AMADID 020793, Agilent Technologies, Santa Clara, CA, USA). The median resolution is about $200 \mathrm{~kb}$, with a 440 -bp resolution in $12.5 \mathrm{Mb}$ of the WBS region (chr7:67558714_79837248, hg19). The array-CGH procedures were performed according to the manufacturer's instructions versus a control DNA. The $244 \mathrm{~K}$ slides were scanned on an Agilent DNA Microarray Scanner, and images were extracted with Feature Extraction software (10.7.3.1). Data analysis was carried out with Agilent Cytogenomics (2.9.2.4). A copy number variation was validated if an abnormal $\log _{2}$ ratio was obtained for at least 3 contiguous oligonucleotides. The array-CGH results were analyzed with the UCSC (University of California, Santa Cruz, CA, USA) hg19 assembly.

Standard G- and R-banded blood karyotypes were determined by standard procedures.

WGS was performed on genomic libraries following the Illumina TruSeq protocol (Illumina, San Diego, CA, USA). Briefly, $3 \mu \mathrm{g}$ of DNA were fragmented by sonication and purified to yield fragments of $350 \mathrm{bp}$. Paired-end adaptor oligonucleotides from Illumina were ligated on repaired A-tailed fragments, then purified, and indexed. DNA library was sequenced on an Illumina NextSeq 500 as paired-end 101-bp reads using the High Output (300 cycles) NextSeq500 kit. Image analysis and base calling were performed using Illumina Real Time Analysis Pipeline 2 with default parameters. The alignment on reference human genome hg19 was performed by BWA/GATK Whole Genome Sequencing workflow V1.6 from BaseSpace (Illumina). SVdetect 21 V0.8b was then used for the rearrangement detection and Integrative Genomics Viewer Broad Institute V2.3 for their visualization. Polymerase chain reactions (PCR) were performed using primer pairs selected on each side of the breakpoint region delimited by WGS (primer sequences available from the authors upon request). Junction fragments were amplified using the Taq DNA Core kit 25 (MP Biomedicals, Solon, OH, USA) according to the following protocol: $120 \mathrm{ng}$ DNA was mixed with $1.75 \mathrm{mM} \mathrm{MgCl}_{2}, 0.2 \mathrm{mM}$ dNTP mix, $0.3 \mathrm{mM}$ forward primer, $0.3 \mathrm{mM}$ reverse primer in a final volume of $50 \mu \mathrm{L}$, and incubated with an initial denaturation for $10 \mathrm{~min}$ at $96^{\circ} \mathrm{C}$, followed by 35 cycles of denaturation for $1 \mathrm{~min}$ at $96^{\circ} \mathrm{C}$, hybridization for 1 min at $60^{\circ} \mathrm{C}$, and elongation for $1 \mathrm{~min}$ at $72^{\circ} \mathrm{C}$, with a final elongation phase for $10 \mathrm{~min}$ at $72^{\circ} \mathrm{C}$. DNA from a negative control who was not a carrier of chromosomal rearrangement was amplified parallel to the patient. DNAs were also amplified with the primer pair for the ATP1A3 gene (exons 7 and 8) as positive control. PCR products were verified on LabChip GX (PerkinElmer, Waltham, 


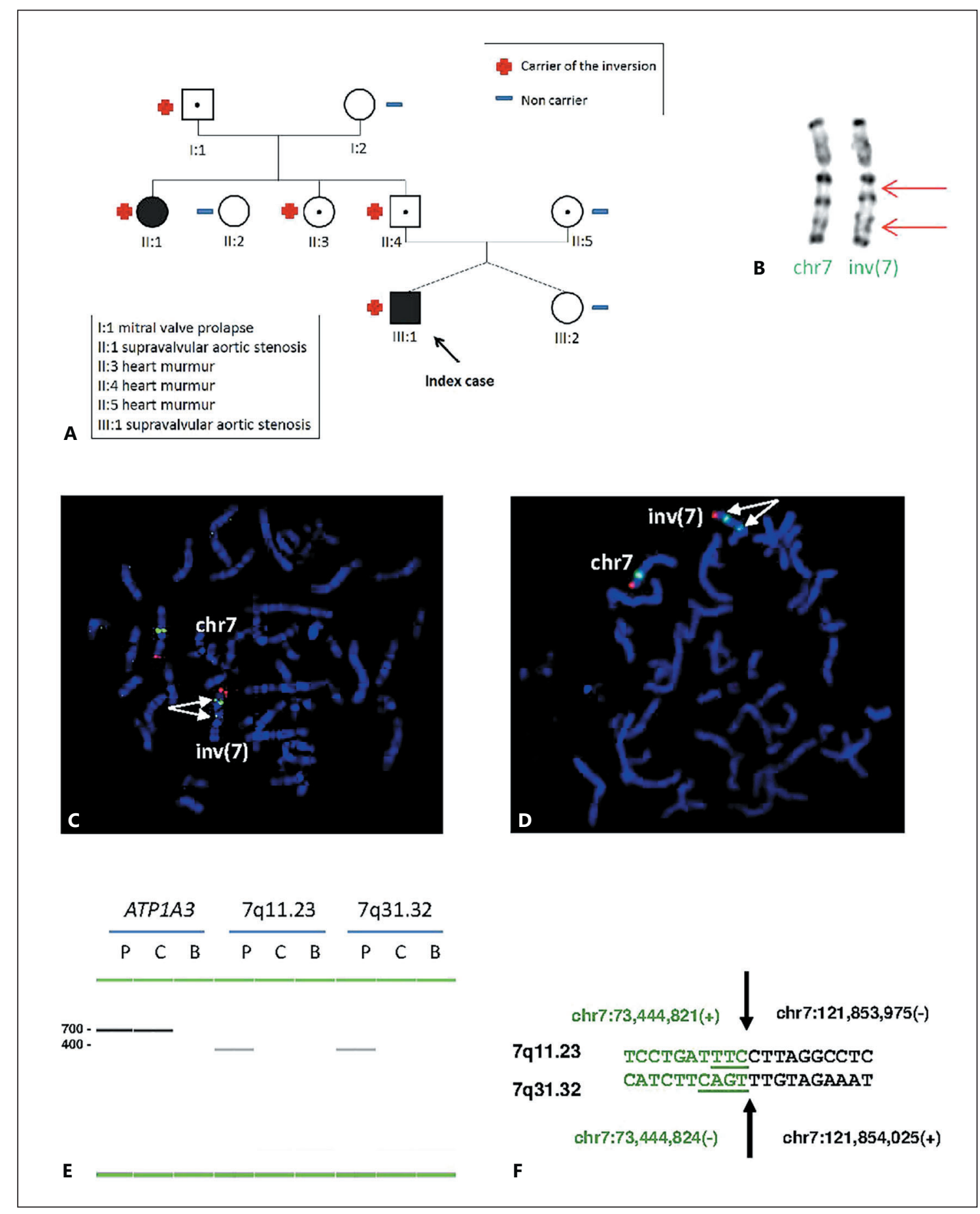

Fig. 1. A Family pedigree. B R-banded chromosome 7 showing a paracentric inversion $\operatorname{inv}(7)(\mathrm{q} 11.2 ; \mathrm{q} 31)$ (arrows). C FISH using RP11-644H24 probe (7q11.23) in green (FITC) and 7qter control probe in red showing a split green signal on derivative chromosome 7 (arrows). D FISH using RP11-560I19 BAC (7q31.32) in green (FITC) and 7qter control probe in red showing a split green signal on derivative chromosome 7 (arrows). E Amplification of junction fragments by PCR and migration on a $2 \%$ agarose gel. P, patient; C, control; B, blank; ATP1A3, positive control; 7q11.23, proximal junction fragment; 7q31.32, distal junction fragment. F Sanger sequencing determining the breakpoints at bp level: sequence of proximal junction fragment (7q11.23) and sequence of distal junction fragment ( $7 \mathrm{q} 31.32)$. The $7 \mathrm{q} 11.23$ sequence is in green, $7 \mathrm{q} 31.32$ is in black. Genomic coordinates are given according to hg19 and strand orientation is indicated (+ or -). Regions of microhomology are underlined. Note that there is a loss of $2 \mathrm{bp}$ at the 7q11.23 breakpoint and of $49 \mathrm{bp}$ at the $7 \mathrm{q} 31.32$ breakpoint. 
Table 1. Cases of ELN disruption in the literature

\begin{tabular}{llllll}
\hline & Curran et al., 1993 & von Dadleszen et al., 2000 & Duba et al., 2002 & Malčić et al., 2008 & Present case \\
\hline Rearrangement & $\mathrm{t}(6 ; 7)(\mathrm{p} 21.1 ; \mathrm{q} 11.23)$ & $\mathrm{t}(6 ; 7)(\mathrm{q} 27 ; \mathrm{q} 11.23)$ & $\mathrm{t}(7 ; 16)(\mathrm{q} 11.23 ; \mathrm{q} 13)$ & $\mathrm{t}(7 ; 14)(\mathrm{q} 11.23 ; \mathrm{p} 12)$ & $\mathrm{inv}(7)(\mathrm{q} 11.23 \mathrm{q} 31.32)$ \\
Inheritance & Familial & De novo & Familial & Familial & Familial \\
$\begin{array}{l}\text { Affected individuals } \\
\text { Clinical features }\end{array}$ & 5 & 1 & 5 & 3 & 5 \\
& SVAS, hoarse voice, & SVAS, hydrops fetalis & Hoarse voice, WBS & SVAS, left ventricular & SVAS, heart murmur, mitral \\
& facial dysmorphism & & & intracavitary stenosis, & valve prolapse \\
& & & & peripheral pulmonary & artery stenosis \\
Characterization & Southern blot & FISH & FISH & FISH & WGS \\
ELN disruption & Exon 28 & Unspecified & Intron 5 & Unspecified & Intron 1 \\
\hline
\end{tabular}

SVAS, supravalvular aortic stenosis; WBS, Williams-Beuren syndrome; WGS, whole-genome sequencing.

MA, USA). Following, specific products corresponding to the junction fragment were sequenced by Sanger method.

FISH was performed using bacterial artificial chromosome (BAC) clones spanning the breakpoint region as defined by the WGS results and selected through the UCSC genome browser. They were commercially available (RP11-644H24; chr7: 73411275_73548266, hg19) (BlueFish, BlueGnome, Cambridge, UK) or FITC (fluorescein isothiocyanate) labeled by nick translation (RP11-560I19; chr7:121856401_121858491, hg19) and hybridized on metaphase spread with appropriate control probe (7qter) (Cytocell, Cambridge, UK).

\section{Results}

For the index case, array CGH with oligonucleotide enrichment at the 7q11.23 locus found no cryptic imbalance. Standard blood karyotyping revealed a balanced paracentric chromosome 7 inversion: $46, X Y, \operatorname{inv}(7)(q 11.2 q 31)$ (Fig. 1B). In order to characterize the effect of this rearrangement on the patient's phenotype, a paired-end WGS was performed. It yielded a $10.9 \times$ physical coverage and made it possible to delineate the breakpoints in chromosome 7 in the 176 -bp region at $7 \mathrm{q} 11.23$ and in the 193 -bp region at 7q31.32. Breakpoints were consistent with cytogenetic findings and were verified by FISH (Fig. 1C, D). Sanger sequencing of junction fragments, amplified by PCR (Fig. 1E), identified the breakpoints at the hg19 positions chr7:73444821_73444824 at 7q11.23 and chr7: 121853975_121854025 at 7q31.32. A 3-bp microhomology with a 2-bp loss was detected at the proximal breakpoint and a 4-bp microhomology with a 49-bp loss at the distal breakpoint (Fig. 1F). The rearrangement disrupted the ELN gene (intron 1) at 7q11.23. No pathogenic gene was involved at the 7q31.32 breakpoint or in its neighborhoods. The balanced paracentric chromosome 7 inversion was inherited from the father and the paternal grandfather, and was shared with 2 paternal aunts including the SVASaffected aunt (Fig. 1A).

\section{Discussion and Conclusion}

Only few cases of ELN disruption have been reported so far. They included 1 prenatal sporadic case [von Dadelszen et al., 2000] and 3 familial cases with multiple affected members [Curran et al., 1993; Duba et al., 2002; Malčić et al., 2008]. The phenotypic spectrum varied widely, even amongst members of the same family (Table 1) (SVAS and other cardiac anomalies, features belonging to the WBS spectrum, such as facial dysmorphism, hoarse voice [Curran et al., 1993; Duba et al., 2002], and intellectual disability [Duba et al., 2002]). It was proposed that elastin contribution to other connective tissues could explain the expanded phenotype as well as a long-range position effect for intellectual disability. However, all these previous cases were only characterized by FISH or Southern blotting, and no array CGH could exclude cryptic chromosomal imbalances. We report the first case of ELN disruption characterized by WGS, providing accurate genomic breakpoint positions and excluding any imbalances. Our family only presented with cardiac features. This supports the fact that precise characterization is necessary to improve the genotype-phenotype correlation in ELN arteriopathy.

WGS has proven to be a powerful tool in determining breakpoints at bp levels. It allows the assessment of gene disruption and the detection of associated cryptic imbalances. It may also contribute to elucidate breakpoint 
mechanisms. In the current case, we observed microhomologies and nucleotide deletions, suggesting microhomology-mediated mechanisms such as MMBIR or FosTes [Ottaviani et al., 2014].

Over the past few years, there has been a tendency to replace karyotype and FISH analyses by array CGH in the first-line diagnosis of congenital malformations and intellectual disability, including WBS. Strategies for molecular diagnosis of ELN arteriopathy rely on Sanger sequencing to identify point mutations or small insertions/ deletions and multiplex ligation probe amplification and real-time quantitative PCR to detect partial or complete exon deletions [Merla et al., 2012]. More recently, panel or exome sequencing have become alternative diagnostic approaches for mutation detection [Poninska et al., 2016; Seidelmann et al., 2017]. Other than FISH, neither of those techniques used in current diagnostic for elastin arteriopathy is able to detect balanced rearrangements susceptible to disrupt ELN. Although ELN gene disruption seems to be rarely involved, it should be kept in mind that a normal array CGH or a Sanger, panel, or exome sequencing result is not sufficient to rule out this hypothesis. With the future perspective of WGS as a diagnostic tool, it will be important to include a structural variation analysis in order to detect balanced rearrangements and gene disruptions that otherwise would be unnoticed.

\section{Acknowledgments}

The authors wish to thank the patient and his family for their kind cooperation, all colleagues who provided help for this report, and the Hospices Civils de Lyon for their support.

\section{Statement of Ethics}

Informed consent was obtained from the family.

\section{Disclosure Statement}

The authors have no conflicts of interest to declare.

\section{Author Contributions}

P. Bouvagnet, M. Bakloul, S. Di Filippo, O. Metton, and J. Mitchell cared for the patient. L. Pons, A. Buisson, N. Chatron, A. Labalme, F. Diguet, P.-A. Rollat-Farnier, D. Sanlaville, and C. Schluth-Bolard performed the genetic analysis. L. Pons wrote the first draft of the manuscript, and all the other authors contributed to the manuscript final form.

\section{References}

Curran ME, Atkinson DL, Ewart AK, Morris CA, Metcalfe K, Rucka AK, Smoot L, Hofstadler G, Leppert MF, Keating MT: The elastin gene is disrupted by a translocation associated with supravalvular aortic stenosis. Cell 73:159-168 (1993).

Duba HC, Doll A, Neyer M, Erdel M, Mann C, et al: The elastin gene is disrupted in a family with a balanced translocation $\mathrm{t}(7 ; 16)$ (q11.23;q13) associated with a variable expression of the Williams-Beuren syndrome. Eur J Hum Genet 10:351-361 (2002).

Malčić I, Dilber D, Kniewald H, Lasan R, Begović D, Jelušić M: Supravalvular aortic stenosis and peripheral pulmonary stenosis in family with balanced translocation $\mathrm{T}(7 ; 14)$ and break point within the elastin gene region. Paediatr Croat 52:77-81 (2008).

- Merla G, Brunetti-Pierri N, Piccolo P, Micale L, Loviglio MN: Supravalvular aortic stenosis: elastin arteriopathy. Circ Cardiovasc Genet 5: 692-696 (2012). Tuzler G, et al: Elastin: mutational spectrum in supravalvular aortic stenosis. Eur J Hum Genet 8:955-963 (2000).

Ottaviani D, LeCain M, Sheer D: The role of microhomology in genomic structural variation. Trends Genet 30:85-94 (2014).

Pober BR: Williams-Beuren syndrome. N Engl J Med 362:239-252 (2010).

Poninska JK, Bilinska ZT, Franaszczyk M, Michalak E, Rydzanicz M, et al: Next-generation sequencing for diagnosis of thoracic aortic aneurysms and dissections: diagnostic yield, novel mutations and genotype phenotype correlations. J Transl Med 14:115 (2016).
Schluth-Bolard C, Labalme A, Cordier MP, Till M, Nadeau G, et al: Breakpoint mapping by next generation sequencing reveals causative gene disruption in patients carrying apparently balanced chromosome rearrangements with intellectual deficiency and/or congenital malformations. J Med Genet 50:144-150 (2013).

- Seidelmann SB, Smith E, Subrahmanyan L, Dykas D, Abou Ziki MD, et al: Application of whole exome sequencing in the clinical diagnosis and management of inherited cardiovascular diseases in adults. Circ Cardiovasc Genet 10:e001573 (2017)

von Dadelszen P, Chitayat D, Winsor EJ, Cohen $\mathrm{H}$, MacDonald C, et al: De novo 46,XX,t(6;7) (q27;q11;23) associated with severe cardiovascular manifestations characteristic of supravalvular aortic stenosis and Williams syndrome. Am J Med Genet 90:270-275 (2000). 